\title{
Análisis de resistencia a la fractura de la cerámica de recubrimiento en la cresta marginal de coronas bilaminares de zirconia Y-TZP con infraestructura convencional y modificada: estudio in vitro
}

\section{Fracture analysis of the veneering ceramic on marginal ridge of bilayer zirconium crowns Y-TZP with conventional and modified infraestructure: in vitrum study}

\section{Análise da resistência à fratura de cerâmica de revestimento na crista marginal de coroas bilamelares de zircônio Y-TZP com infraestrutura convencional e modificada: estudio in vitro}

\author{
Diego Alfonso Arcos Bronenberg iD \\ Vinicius Rizzo Marques iD \\ Emerson Flamarion da Cruz iD \\ Endereço para correspondência: \\ Diego Alfonso Arcos Bronenberg \\ Faculdade do Centro Oeste Paulista - FACOP \\ Rua Luiz Gimenez Mocegose, 72 \\ Distrito Industrial \\ 17499-010 - Piratininga - São Paulo - Brasil \\ E-mail: diegoarcosbronenberg@gmail.com
}

Recebido: 06.08.2020

Modificado: 24.08.2020

Aceito: 01.09.2020

\section{RESUMEN}

Las coronas bilaminares de zirconia representan una alternativa de alta estética para la odontología restauradora. Sin embargo, el eslabón débil de este tipo de restauraciones es el despostillamiento y la delaminación de la cerámica de recubrimiento. Siguiendo la hipótesis de que al dar un mayor soporte a la cerámica esta va a tener un mejor comportamiento frente a estas situaciones, se realizó un estudio in vitro de cargas axiales de fuerza hasta el fracaso sobre 20 coronas bilaminares elaboradas con dos tipos de infraestructura: 10 convencionales $(0.8 \mathrm{~mm}$ de espesor uniforme) y 10 modificadas ( $1 \mathrm{~mm}$ de espesor y $2.5 \mathrm{~mm}$ de altura en lingual y proximal) cementadas sobre dados de resina. El valor promedio de las coronas con infraestructura convencional fue de $611.3 \mathrm{~N}$ (D.S. 191.2 N) y de las coronas con infraestructura modificada fue de $727.5 \mathrm{~N}$ (D.S. $173.7 \mathrm{~N}$ ). Se realizaron pruebas paramétricas de los datos obtenidos y T-Student como prueba de comparación entre dos grupos demostrando que, las coronas bilaminares de zirconia con infraestructura modificada tienen un $15 \%$ más de resistencia a la fractura, por lo tanto, la hipótesis nula fue aceptada.

PALABRAS CLAVE: Cerámica. Resistencia flexional. Coronas. 
Análisis de resistencia a la fractura de la cerámica de recubrimiento en la cresta marginal de coronas bilaminares de zirconia Y-TZP con infraestructura convencional y modificada: estudio in vitro

\begin{abstract}
Zirconium bilayered crowns are an excellent alternative in esthetic restorative dentistry. Nevertheless, chipping and delamination is the weakest link in this type of restorations. Considering the hypothesis that giving a better support to the layering ceramic is going to improve its performance in this matter, and in vitro study of continuous axial load until failure applied to 20 bilayer zirconium crowns with two different core designs: 10 conventional $(0.8$ uniform thickness), 10 modified (1 mm thickness and $2.5 \mathrm{~mm}$ height in lingual and proximal areas) cemented on resin dies was done. Mean value for crowns with conventional core is 611.3 N (S.D. 191.2 N) and for modified core is $727.5 \mathrm{~N}$ (S.D. 173.7 N). Parametric tests and T-Student comparison was used to analyze data. Results are that bilayered zirconium crowns with modified core had $15 \%$ more fracture resistance than with conventional core accepting the null hypothesis.
\end{abstract}

KEYWORDS: Ceramics. Flexural strength. Crowns.

\title{
RESUMO
}

As coroas bilaminares de zircônia representam uma alternativa altamente estética para a odontologia restauradora. No entanto, o ponto fraco nesses tipos de restaurações é o lascamento e a delaminação da cerâmica de estratificação. Partindo da hipótese de que dando maior sustentação à cerâmica ela terá um melhor comportamento nestas situações, foi realizado um estudo in vitro das cargas de força axial até a ruptura em 20 coroas bilaminares confeccionadas com dois tipos de infraestrutura: 10 convencionais (espessura uniforme de $0.8 \mathrm{~mm}$ ) e 10 modificados (espessura de $1 \mathrm{~mm}$ e altura de $2.5 \mathrm{~mm}$ em lingual e proximal) cimentados em matrizes de resina. $\mathrm{O}$ valor médio das coroas com infraestrutura convencional foi $611.3 \mathrm{~N}$ (D.P. 191.2 N) e das coroas com infraestrutura modificada foi $727.5 \mathrm{~N}$ (D.P. 173.7 N). Foram realizados testes paramétricos dos dados obtidos e T-Student como teste de comparação entre dois grupos, mostrando que coroas bilamelares de zircônia com infraestrutura modificada apresentam $15 \%$ mais resistência à fratura, portanto a hipótese nula foi aceita.

PALAVRAS-CHAVE: Cerâmica. Resistência à flexão. Coroas. 


\section{INTRODUCCIÓN}

Debido al constante cambio y demandas estéticas de las sociedades y por consiguiente de los profesionales clínicos, la industria no cesa en la búsqueda de nuevas tecnologías de desarrollo, mejoramiento de materiales que posean una mayor bio compatibilidad, longevidad, sellado marginal, resistencia, función y estética.

Dentro del tipo de cerámicas policristalinas, se encuentra la zirconia policristalina tetragonal estabilizada con óxido de itrio (Y-TZP Yttrium Stabilizaed Tetragonal Zirconia Polycrystal), introducida en el campo de la rehabilitación oral para solucionar los problemas de baja resistencia a la flexión y compresión que presentan otro tipo de cerámicas libres de metal. Estas poseen una resistencia a la flexión de $1200 \mathrm{MPa}$ y a la fractura de $6 \mathrm{a} 9 \mathrm{MPa}^{1}$.

Se pueden fabricar coronas monolíticas y bilaminares de Y-TZP. Para estas últimas se confeccionan infraestructuras básicamente con dos tipos de diseño: convencionales, las cuales tienen un espesor uniforme de $0.5-0.8 \mathrm{~mm} \mathrm{y}$, modificadas que tienen una cinta lingual de $1 \mathrm{~mm}$ de espesor y $2.5 \mathrm{~mm}$ de alto extendiéndose a las caras proximales ${ }^{1}$.

Debido a la naturaleza opaca de la zirconia y al requerimiento de alta estética, están indicadas las coronas bilaminares, las cuales necesitan de una cobertura de cerámica feldespática con alto contenido de matriz vítrea o sintéticas con base de fluorapatita que posean translucidez, estabilidad de color y brillo.

Sin embargo, está reportado que la fractura de la cerámica de recubrimiento es la mayor causa del fracaso de este tipo de restauraciones ${ }^{1-5}$.

Los rangos de fractura están entre el $5-9 \%$ en 24 a 36 meses $^{5}$, o del 6 y $15 \%$ en un período de 3 a 5 años . $^{6}$.

Son varios los factores que afectan adversamente la resistencia a la fractura de las restauraciones con infraestructura de zirconia: inadecuado diseño de la infraestructura, fragilidad de la cerámica de recubrimiento, daño de la cerámica después de realizar ajustes oclusales, diferencia entre el coeficiente de expansión térmica entre la infraestructura y la cerámica de recubrimiento, bajo coeficiente de difusión térmico de la zirconia, envejecimiento térmico y mecánico, mala adhesión entre la infraestructura y la cerámica de cobertura ${ }^{1,7}$.

Para reducir o eliminar uno de los factores que producen el despostillamiento y delaminación de la cerámica de recubrimiento sobre las infraestructuras de zirconia, se ha creado la hipótesis de que, con un cambio de diseño en la infraestructura, de convencional a modificada, habría una mejora significativa de la longevidad y éxito de este tipo de restauraciones debido a un mayor y mejor soporte para la cerámica de recubrimiento ${ }^{2}$.

El objetivo de esta investigación fue comprobar si se acepta o no la hipótesis nula propuesta la cual es: el valor medio de fuerza de carga axial estática $(\mathrm{N})$ necesario para provocar la fractura de la cerámica de recubrimiento de las coronas con infraestructura modificada es al menos el 15\% más alta que la media de fuerza de carga axial estática para provocar la fractura de la cerámica de recubrimiento de las coronas con infraestructura convencional; mediante un estudio in vitro que consiste en aplicar cargas axiales estáticas incrementales de fuerza continua hasta la fractura a 10 coronas bilaminares de zirconia Y-TZP con infraestructura convencional de un espesor uniforme de $0.8 \mathrm{~mm}$ y 10 coronas bilaminares de zirconia Y-TZP con infraestructura modificada con una cinta lingual de $1 \mathrm{~mm}$. de espesor y 2.5 $\mathrm{mm}$ de alto extendiéndose hacia las caras proximales.

\section{MATERIAL Y MÉTODOS}

El primer molar inferior derecho artificial de ivorina fue preparado para una corona unitaria siguiendo las indicaciones para una restauración totalmente cerámica de zirconia de acuerdo a la técnica de tallado de Magalhães de Padua y Franca Teles utilizando el kit de fresas para preparación de restauraciones (Brasseler, USA) : chaflán a lo largo de la línea cervical entre 1 y $1.5 \mathrm{~mm}$, ángulos redondeados, espacio inter oclusal de $1.5 \mathrm{a} 2 \mathrm{~mm}$, paredes axiales con $8^{\circ}$ a $10^{\circ}$ grados de angulación y 1 a $1.5 \mathrm{~mm}$ de espesor, paredes lisas. ${ }^{1}$.

El diente preparado fue espolvoreado con ZFX Scan Spray (Zimmer Biomed, Alemania) para el escaneado con el scanner ZFX Evolution Plus (Zimmer Biomed, Alemania). La imagen 3D obtenida fue procesada y almacenada por el software ZFX Cad (Zimmer Biomed, Alemania) y transferida a la unidad de maquinado ZFX Mill in House 5x. (Zimmer Biomed, Alemania) para la elaboración de 20 dados de trabajo de resina VeroDentPlus Med 690 (Stratasys, USA) en la impresora Object 260 Dental Selection (Stratasys, USA) para crear un escenario clínico realístico para estudio in vitro ${ }^{2}$ sobre los cuales se cementarán las coronas bilaminares de zirconia.

De la imagen del diente preparado se diseñó con el software ZFX Cad, dejando un espacio de $80 \mathrm{~m}$ para la capa de cemento, una infraestructura convencional de $0.8 \mathrm{~mm}$ de espesor uniforme y una infraestructura modificada con una cinta lingual de $1 \mathrm{~mm}$ de espesor y $2.5 \mathrm{~mm}$ de alto extendiéndose a las caras proximales con un espesor de 0.5 $\mathrm{mm}^{2}$. La información de cada uno de los diseños fue enviada a la unidad de maquinado ZFX Mill in House 5x para fabricar 10 unidades convencionales y 10 unidades modificadas de zirconia pre sinterizada Katana ML (Kuraray, Noritake Japón).

El proceso de sinterización se terminó en el horno Dekema Austromat 664 (Dekema, Austria) con el programa de $10^{\circ} \mathrm{C}$ por minuto hasta alcanzar una temperatura de 
Análisis de resistencia a la fractura de la cerámica de recubrimiento en la cresta marginal de coronas bilaminares de zirconia Y-TZP con infraestructura convencional y modificada: estudio in vitro

$1.500^{\circ} \mathrm{C}$. Se mantuvo a esa temperatura por dos horas y se empezó el proceso de enfriamiento de $10^{\circ} \mathrm{C}$ por minuto hasta llegar a temperatura ambiente.

Se inspeccionaron cada una de las infraestructuras y se realizó el ajuste necesario para el perfecto asentamiento en los dados de trabajo.

Para estandarizar la anatomía, contorneo y espesor de la cerámica de recubrimiento de las 20 unidades, se elaboró previamente a la preparación del primer molar inferior derecho, una matriz de silicona (Zhermack, Italia) que sirva como guía.

La cerámica feldespática con leucita de recubrimiento, la cual posee un coeficiente de $92.7 \mathrm{Mpa}$ de resistencia a la fractura y glaseado final CERABIEN (Kuraray, Noritake Japón) fue aplicada por un laboratorista altamente calificado sobre cada una de las infraestructuras con la técnica por capas cocinándolas en el horno Dekema Autromat 624 (Dekema, Austria) siguiendo las exactas especificaciones técnicas de la compañía utilizando la matriz de silicona anteriormente mencionada para estandarizar la forma final de cada una de las coronas. Para el enfriamiento de cada una de las capas se utilizó la técnica de proceso lento de enfriamiento para así reducir los efectos de diferencia entre los coeficientes de expansión y difusión térmica existente entre la infraestructura y la cerámica de recubrimiento ${ }^{8}$.

Las 20 coronas terminadas se separaron en 2 grupos de 10 especímenes cada uno, G1: infraestructura convencional; G2: infraestructura modificada.

Todas las coronas fueron tratadas su superficie interna previa cementación con un sandblast de óxido de aluminio de $50 \mathrm{~m}$ (Renfert, Alemania) a 2.5 bar de presión por 15 segundos a una distancia de 10 a $15 \mathrm{~cm}^{1}$.

Los 20 dados de trabajo de resina VeroDentPlus Med 690 (Stratasys, USA) fueron sumergidos y almacenados por 30 días en un acuario con termostato (Ortoart, Brasil), lleno de agua destilada a una temperatura de $37^{\circ} \mathrm{C}$ para lograr así una hidratación completa y eliminar cualquier efecto de expansión dimensional por absorción de agua ${ }^{2}$.

Para la cementación, a todas las coronas se les realizó una limpieza en ultrasonido por 60 segundos (Codyson, China), secado con aire limpio y luego se cementaron sobre los dados de trabajo de resina VeroDentPlus Med 690 (Stratasys, USA) utilizando un cemento resinoso autoadhesivo SoloCem (Coltene, Switzerland) aplicando una presión constante de $10 \mathrm{~N}^{2}$ con un peso metálico previamente comprobado por dos minutos siguiendo las especificaciones del fabricante.

Una vez cementadas las coronas en los dados de trabajo, se sumergieron nuevamente en el acuario con agua destilada a $37^{\circ} \mathrm{C}$ por 7 días para así proveer de un adecuado equilibrio de agua antes de realizar el experimento ${ }^{2}$.

Una vez terminado todo el proceso, se obtuvo el siguiente grupo de estudio.
Tabla 1 - Grupo de estudio de coronas con infraestructura convencional y modificada.

\begin{tabular}{|c|c|c|c|c|c|c|c|}
\hline GRUPO & $\begin{array}{c}\text { TECNICADE } \\
\text { APLICACAON DE } \\
\text { LACERAMICA } \\
\text { DE } \\
\text { RECUBRIMIENT } \\
0\end{array}$ & $\begin{array}{l}\text { MATERIAL DE } \\
\text { INFRAESTRUCT } \\
\text { URA }\end{array}$ & $\begin{array}{c}\text { TPPO DE } \\
\text { INFRAESTRUCT } \\
\text { URA }\end{array}$ & $\begin{array}{l}\text { MARCADE } \\
\text { INFRAESTRUCT } \\
\text { URA }\end{array}$ & $\begin{array}{c}\text { CERAMICADE } \\
\text { RECUBRIMIENT } \\
0\end{array}$ & $\begin{array}{c}\text { TIPO DE } \\
\text { CERAMICADE } \\
\text { RECUBRIMIENT } \\
\text { O }\end{array}$ & $\begin{array}{c}\text { MARCA } \\
\text { CERAMICADE } \\
\text { RECUBRIMIENT } \\
O\end{array}$ \\
\hline $\begin{array}{c}\text { CONVENCIONAL } \\
\text { G:1 }\end{array}$ & $\begin{array}{l}\text { TECNIAAPOR } \\
\text { CAPAS }\end{array}$ & $\begin{array}{l}\text { KATANA ML PRE } \\
\text { SINTERIZADA }\end{array}$ & YTZP & $\begin{array}{l}\text { KURARAY } \\
\text { NORITAKE }\end{array}$ & CERABIEN & $\begin{array}{l}\text { CERAMICADE } \\
\text { VIDRID } \\
\text { FELDESPATICA }\end{array}$ & $\begin{array}{l}\text { KURARAY } \\
\text { NORITAKE }\end{array}$ \\
\hline $\begin{array}{c}\text { MODIFICADA } \\
\text { G:2 }\end{array}$ & $\begin{array}{c}\text { TECNICA POR } \\
\text { CAPAS }\end{array}$ & $\begin{array}{l}\text { KATANA ML PRE } \\
\text { SINTERIZADA }\end{array}$ & YTZP & $\begin{array}{l}\text { KURARAY } \\
\text { NORITAKE }\end{array}$ & CERABIEN & $\begin{array}{l}\text { CERAMICADE } \\
\text { VIDRID } \\
\text { FELDESPATICA }\end{array}$ & $\begin{array}{l}\text { KURARAY } \\
\text { NORITAKE }\end{array}$ \\
\hline
\end{tabular}

Cada uno de los especímenes fue colocado en una máquina de comprobación universal (MTS, USA) en posición vertical con un sujetador estándar. Se aseguró un contacto de tres puntos entre el indentador y cada corona en la cresta marginal mesial antes de ser sometidas a las cargas de fuerza. Se aplicó fuerzas estáticas de presión hasta el fracaso utilizando un indentador esférico metálico de 3.7 $\mathrm{mm}$ de diámetro a una velocidad cruceta de $0.5 \mathrm{~mm} / \mathrm{min}^{1}$.

Cuatro modos de fracaso fueron identificados: fracaso cohesivo de la cerámica de recubrimiento, fracaso de adhesión entre la cerámica de recubrimiento y la infraestructura, fracaso de la infraestructura y fracaso de la base.

Los datos arrojados de cada una de las muestras (carga de fracaso en $\mathrm{N}$ ) fueron anotados y tabulados en una tabla de datos brutos para poder realizar el análisis estadístico correspondiente.

La metodología estadística utilizada para el análisis fue primeramente establecer si los datos provienen de una distribución normal para de acuerdo a ello establecer qué tipo de pruebas (paramétricas o no paramétricas) vamos a usar. Para lograr esto, debido a que tenemos 10 datos o menos en cada uno de los grupos, aplicamos la prueba de Shapiro-Wilk (se utiliza cuando es menor a 50 datos) a través del programa SPSS (SPSS 23.0, Inc., Chicago, IL) obteniendo los siguientes resultados.

Tabla 2 - Prueba de Shapiro-Wilk

\begin{tabular}{ccccc}
\hline Observación & Muestras & Estadístico & gl & Sig. \\
\hline Valor De Carga De Fractura (N) & Convencional & 0.965 & 10 & 0.843 \\
& Modificada & 0.935 & 10 & 0.503 \\
Fracaso Cohesivo (N) De La Cerámica & Convencional & 0.893 & 7 & 0.291 \\
De Recubrimiento & Modificada & 0.984 & 5 & 0.953 \\
Fracaso Cohesivo (N) Entre La & Convencional & 0.877 & 4 & 0.326 \\
$\begin{array}{c}\text { Cerámica De Recubrimiento Y La } \\
\text { Infraestructura }\end{array}$ & Modificada & 0.994 & 3 & 0.856 \\
Fracaso Infraestructura (N) & Convencional & & & \\
& Modificada & 0.994 & 3 & 0.856 \\
Fracaso De La Base & Convencional & 0.812 & 3 & 0.144 \\
& Modificada & 0.843 & 6 & 0.139 \\
\hline \hline
\end{tabular}

El nivel de significancia que se obtuvo en cada una de las pruebas fue mayor que $\alpha=0.05$. A partir de este resultado podemos afirmar que todas las muestras se comportan de manera normal.

Siguiendo el protocolo estadístico, se sacaron la media, desviación estándar, valor mínimo y máximo de los datos de 
carga de fractura de cada uno de los grupos correspondientes a cada modo de fracaso. Debido a que pertenecen a una población con distribución normal, son muestras independientes, se realizó comparación entre dos grupos, se realizaron pruebas paramétricas T-Student (tamaño de muestra diferente y varianzas iguales) para aceptar o rechazar la hipótesis nula o alternativa las cuales son:

Ho: El valor medio de fuerza de carga axial estática $(\mathrm{N})$ necesario para provocar la fractura de la cerámica de recubrimiento de las coronas con infraestructura modificada es al menos el 15\% más alta que la media de fuerza de carga axial estática para provocar la fractura de la cerámica de recubrimiento de las coronas con infraestructura convencional.

Ha: El valor medio de fuerza de carga axial estática $(\mathrm{N})$ necesario para provocar la fractura de la cerámica de recubrimiento de las coronas con infraestructura modificada es menor al $15 \%$ que la media de fuerza de carga axial estática para provocar la fractura de la cerámica de recubrimiento de las coronas con infraestructura convencional.

Segundo, dividimos en cuatro grupos los diferentes modos de fracaso causados por la carga axial estática de fractura sobre las coronas con infraestructura convencional y modificada para poder realizar las comparaciones entre ellas:

- Comparación entre las Muestras Convencional y Modificada en Valor de Carga de Fractura.

- Comparación entre las Muestras Convencional y Modificada en Fracaso Cohesivo de la Cerámica de Recubrimiento.

- Comparación entre las Muestras Convencional y Modificada en Fracaso Adhesivo entre la Cerámica de Recubrimiento y la Infraestructura.

- Comparación entre las Muestras Convencional y Modificada en Fracaso de la Base.

- NOTA: En el modo de fracaso de la infraestructura, no se pudo hacer comparación y prueba de hipótesis debido a que solo las muestras modificadas presentaron este tipo de fractura.

Para cada uno de estos grupos realizamos la prueba de hipótesis anteriormente mencionada, siguiendo el siguiente esquema:

1. Construimos una hoja de cálculo que nos permita probar diferentes escenarios.

2. Probamos si la media de la muestra de coronas modificadas soporta un $15 \%$ más de carga que las coronas convencionales.

3. En los casos en los que la estructura modificada soportaba un 15\% más de carga (TODOS), probamos de manera ascendente $(+5 \%)$ en cada ocasión hasta encontrar el punto en el que la hipótesis nula debía ser rechazada.

4. Una vez que llegamos al punto de rechazo probamos de manera descendente $(-1 \%)$ hasta encontrar el porcentaje en el que la hipótesis nula se aceptaba. Este punto fue diferente para cada uno de los grupos.

5. Finalmente presentamos los resultados del 15\% de mejora y del punto máximo de mejora para cada uno de los grupos.
Para realizar las pruebas estadísticas, utilizamos la siguiente nomenclatura:

$\mu_{1}$ : Media poblacional de estructura convencional

$\mu_{2}$ : Media poblacional de estructura modificada

$n_{1}:$ Tamaño de muestra de estructura convencional

$n_{2}:$ Tamaño de muestra de estructura modificada

$\bar{x}_{1}:$ Media muestral de estructura convencional

$\bar{x}_{2}:$ Media muestral de estructura modificada

$s_{1}$ : Desviación estándar muestral de estructura convencional

$s_{2}$ : Desviación estándar muestral de estructura modificada

$s_{p}^{2}:$ Estimador combinado para varianzas desconocidas

$\Delta_{x \%}:$ Mejora del $x \%$

\section{RESULTADOS}

\section{Comparación de la Muestras en Valor de Carga de Fractura}

\begin{tabular}{cccccc}
\multicolumn{7}{c}{ VALOR DE CARGA DE FRACTURA (N) } \\
\hline & $\mathrm{n}$ & Media & $\begin{array}{c}\text { Desviación } \\
\text { estándar }\end{array}$ & Mínimo & Máximo \\
\hline CONVENCIONAL & 10 & .611 .3 & 191.211 & 341 & 968 \\
MODIFICADA & 10 & 727.5 & 173.717 & 455 & 963 \\
\hline \hline
\end{tabular}

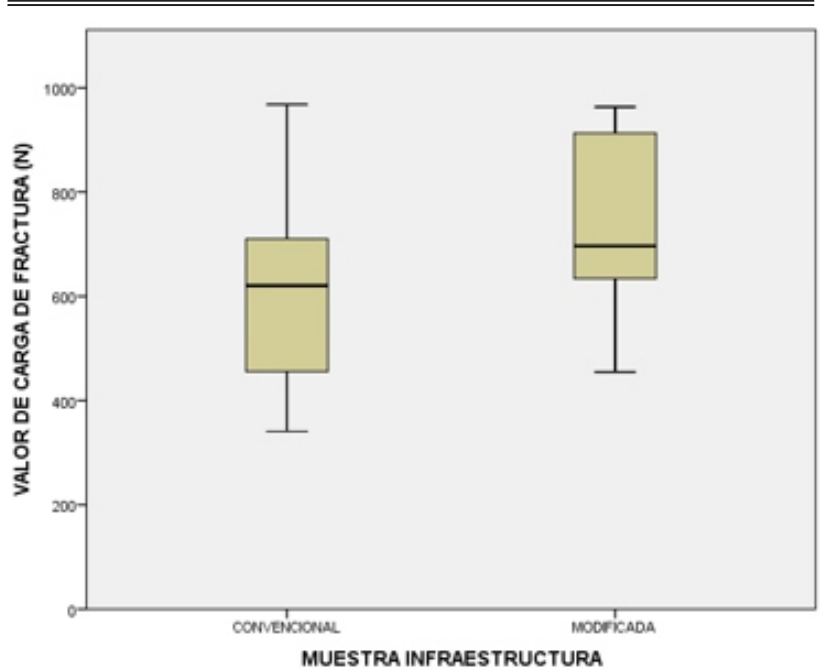

Figura 1 - Comparación de medias del valor de carga de fractura entre muestras convencional y modificada. 
Análisis de resistencia a la fractura de la cerámica de recubrimiento en la cresta marginal de coronas bilaminares de zirconia Y-TZP con infraestructura convencional y modificada: estudio in vitro

\section{Comparación de la Muestras en Fracaso Cohesivo de la Cerámica de Recubrimiento}

\begin{tabular}{cccccc}
\multicolumn{7}{c}{ VALOR DE CARGA DE FRACTURA (N) } \\
\hline & $\mathrm{n}$ & Media & $\begin{array}{c}\text { Desviación } \\
\text { estándar }\end{array}$ & Mínimo & Máximo \\
\hline CONVENCIONAL & 7 & 578.29 & 224.13 & 341 & 968 \\
MODIFICADA & 5 & 661.60 & 176.705 & 455 & 913 \\
\hline \hline
\end{tabular}

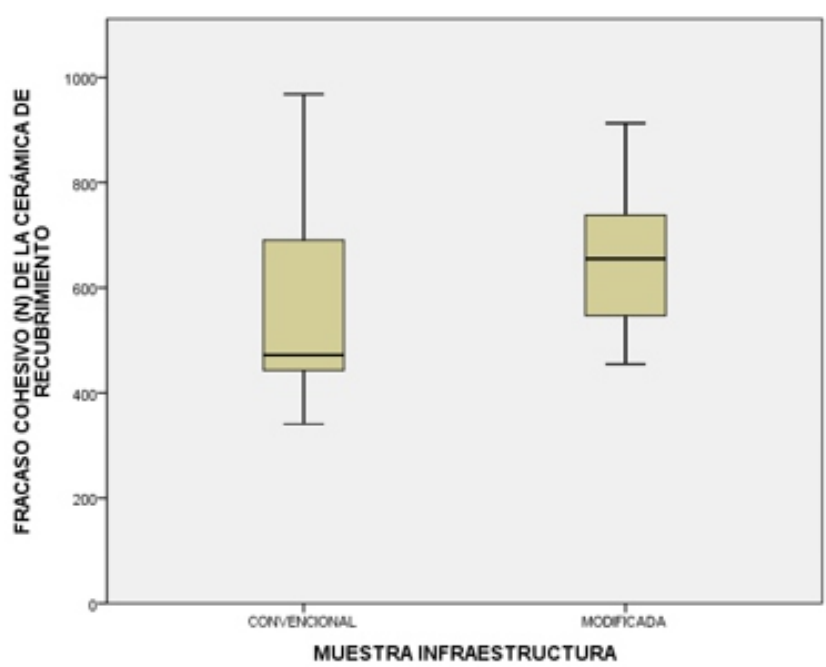

Figura 2 - Comparación de medias del fracaso cohesivo (N) de muestras convencionales y modificadas.

Comparación de la Muestras en Fracaso Adhesivo entre la Cerámica de Recubrimiento y la Infraestructura

\begin{tabular}{cccccc}
\multicolumn{7}{c}{ VALOR DE CARGA DE FRACTURA (N) } \\
\hline & $\mathrm{n}$ & Media & $\begin{array}{c}\text { Desviación } \\
\text { estándar }\end{array}$ & Mínimo & Máximo \\
\hline CONVENCIONAL & 4 & 661.00 & 262.219 & 430 & 968 \\
MODIFICADA & 3 & 646.67 & 95.772 & 547 & 738 \\
\hline \hline
\end{tabular}

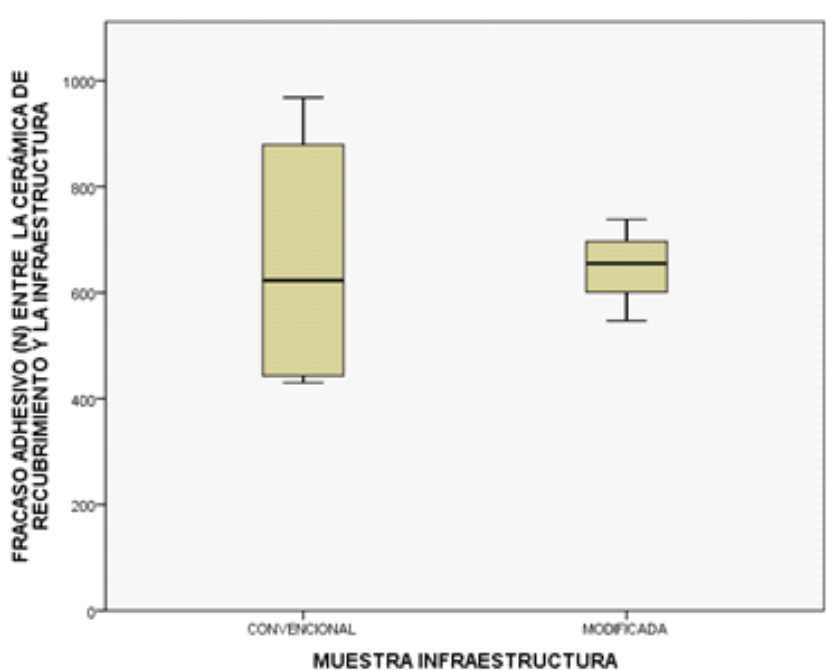

Figura 3 - Comparación de medias del fracaso adhesivo (N) y la Infraestructura de muestras convencional y modificada.
Comparación de la Muestras en Fracaso de la Base

\begin{tabular}{lccccc}
\multicolumn{7}{c}{ VALOR DE CARGA DE FRACTURA (N) } \\
\hline & $\mathrm{n}$ & Media & $\begin{array}{c}\text { Desviación } \\
\text { estándar }\end{array}$ & Mínimo & Máximo \\
\hline CONVENCIONAL & 3 & 688.33 & 33.292 & 650 & 710 \\
MODIFICADA & 6 & 813.33 & 151.784 & 634 & 963 \\
\hline \hline
\end{tabular}

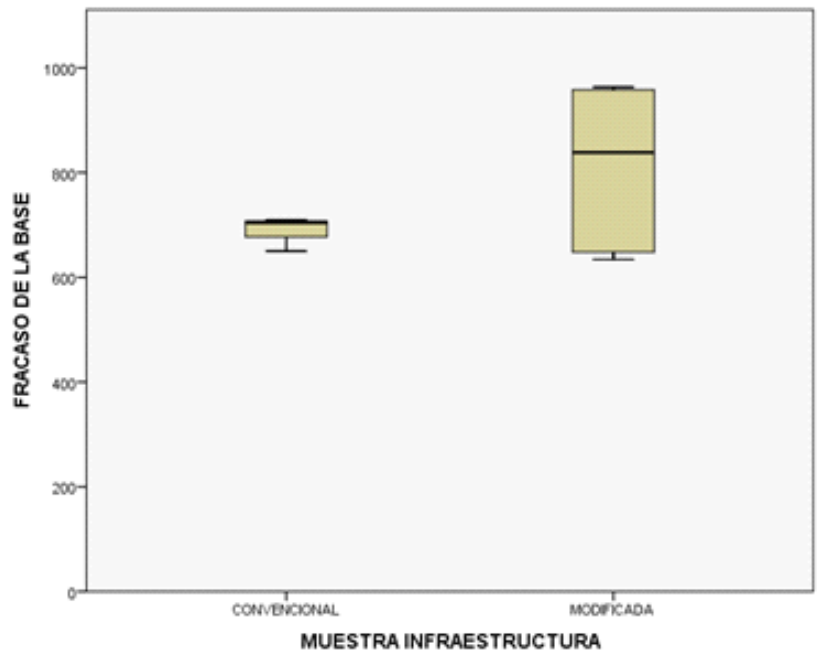

Figura 4 - Porcentaje de fracaso de la Base en muestras convencionales y modificadas.

\section{Fracaso de Infraestructura}

\begin{tabular}{cccccc}
\multicolumn{7}{c}{ VALOR DE CARGA DE FRACTURA (N) } \\
\hline & $\mathrm{n}$ & Media & $\begin{array}{c}\text { Desviación } \\
\text { estándar }\end{array}$ & Mínimo & Máximo \\
\hline CONVENCIONAL & 0 & 0.00 & 0.00 & 0.00 & 0.00 \\
MODIFICADA & 3 & 646.67 & 95.77 & 547 & 738 \\
\hline \hline
\end{tabular}

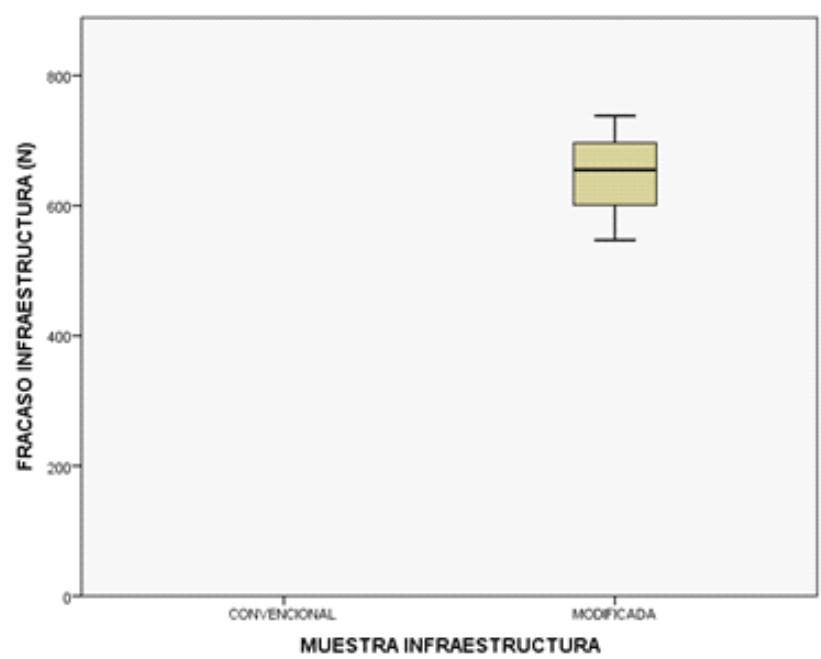

Figura 5 - Porcentaje de fracaso de la infraestructura en muestras convencionales y modificadas.

\section{Comparación de la Muestras en Valor de Carga de Fractura}


Primero compararemos las muestras para una mejora del 15\% en relación con la estructura convencional.

Tabla 3 - Valor de carga de fractura en muestras convencional y modificada.

\begin{tabular}{cccccc}
\multicolumn{7}{c}{ VALOR DE CARGA DE FRACTURA (N) } \\
\hline & $\mathrm{n}$ & Media & $\begin{array}{c}\text { Desviación } \\
\text { estándar }\end{array}$ & Minimo & Máximo \\
\hline CONVENCIONAL & 10 & 611.3 & 191.211 & 341 & 968 \\
MODIFICADA & 101 & 727.5 & 173.717 & 455 & 963 \\
\hline \hline
\end{tabular}

$\Delta_{15 \%}: 611.3 * 0.15=91.7$

$$
\begin{aligned}
& H_{0}: \mu_{1}-\mu_{2} \leq-91.7 \\
& H_{a}: \mu_{1}-\mu_{2}>-91.7 \\
\alpha= & 0.05(95 \% \text { de confianza })
\end{aligned}
$$

El estadístico de prueba:

$\mathrm{t}_{\mathrm{o}}=-0.899$

Dado que $-0.899<2.101$ se acepta $H_{\circ}$

Segundo, compararemos las muestras para una mejora del $29 \%$ en relación com la

$$
\begin{aligned}
\Delta_{2996}: 611.3 * 0.29=177.27 & \\
& H_{0}: \mu_{1}-\mu_{2} \leq-177.27 \\
& H_{a}: \mu_{1}-\mu_{2}>-177.27 \\
\alpha & =0.05(95 \% \text { de confianza })
\end{aligned}
$$

El estadístico de prueba:

$$
\mathrm{t}_{\mathrm{o}}=2.242
$$

Dado que $2.242>2.101$ se rechaza $H_{o}$.

Podemos decir que las coronas con estructura modificada soportan entre un $15 \%$ y $28 \%$ más de fuerza que las coronas con estructura convencional.

\section{Comparación de la Muestras en Fracaso Cohesivo de la Cerámica de Recubrimiento}

Primero compararemos las muestras para una mejora del $15 \%$ en la relación estructura convencional.

Tabla 4 - Fracaso cohesivo en muestras convencional y modificada.

\begin{tabular}{cccccc}
\multicolumn{7}{c}{ VALOR DE CARGA DE FRACTURA (N) } \\
\hline & $\mathrm{n}$ & Media & $\begin{array}{c}\text { Desviación } \\
\text { estándar }\end{array}$ & Minimo & Máximo \\
\hline CONVENCIONAL & 7 & 578.29 & 224.13 & 341 & 968 \\
MODIFICADA & 5 & 661.60 & 176.705 & 455 & 913 \\
\hline \hline
\end{tabular}

$\Delta_{15 \%}: 578.29 * 0.15=86.7$

$$
\begin{aligned}
& H_{0}: \mu_{1}-\mu_{2} \leq-86.7 \\
& H_{a}: \mu_{1}-\mu_{2}>-86.7 \\
\alpha= & 0.05(95 \% \text { de confianza })
\end{aligned}
$$

El estadístico de prueba:

$\mathrm{t}_{\mathrm{o}}=0.064$

Dado que $0.064<2.228$ se acepta $H_{\text {o }}$

Segundo, compararemos las muestras para una mejora del $35 \%$ en relación com la estructura convencional.

$$
\begin{aligned}
\Delta_{35 \%}: 578.29 * 0.35=202.4015 \\
\\
H_{0}: \mu_{1}-\mu_{2} \leq-202.4015 \\
\\
H_{a}: \mu_{1}-\mu_{2}>-202.4015 \\
\alpha=0.05(95 \% \text { de confianza })
\end{aligned}
$$

El estadístico de prueba:

$$
\mathrm{t}_{\mathrm{o}}=2.253
$$

Dado que $2.253>2.228$ se rechaza $H_{\mathrm{o}}$.

Podemos decir que las coronas con estructura modificada soportan entre un $15 \%$ y $34 \%$ más de fuerza que las coronas con estructura convencional.

\section{Comparación de la Muestras en Fracaso Adhesivo entre la Cerámica de Recubrimiento y la Infraestructura}

Primero compararemos las muestras para una mejora del $15 \%$ en relación con la estructura convencional.

Tabla 5 - Fracaso adhesivo en muestras convencional y modificada.

\begin{tabular}{cccccc}
\multicolumn{7}{c}{ VALOR DE CARGA DE FRACTURA (N) } \\
\hline & $\mathrm{n}$ & Media & $\begin{array}{c}\text { Desviación } \\
\text { estándar }\end{array}$ & Mínimo & Máximo \\
\hline CONVENCIONAL & 4 & 661.00 & 262.219 & 430 & 968 \\
MODIFICADA & 3 & 646.67 & 95.772 & 547 & 738 \\
\hline \hline
\end{tabular}

$\Delta_{1596}: 661 * 0.15=99.15$

$$
\begin{gathered}
H_{0}: \mu_{1}-\mu_{2} \leq-99.15 \\
H_{a}: \mu_{1}-\mu_{2}>-99.15 \\
\alpha=0.05(95 \% \text { de confianza })
\end{gathered}
$$

El estadístico de prueba:

$$
\mathrm{t}_{\mathrm{o}}=1.19
$$

Dado que $1.19>2.571$ se acepta $H_{o}$.

Segundo, compararemos las muestras para una mejora del $35 \%$ en relación con la estructura convencional.

$\Delta_{35 \%}: 661 * 0.35=231.35$

$$
\begin{gathered}
H_{0}: \mu_{1}-\mu_{2} \leq-231.35 \\
H_{a}: \mu_{1}-\mu_{2}>-231.35 \\
\alpha=0.05(95 \% \text { de confianza })
\end{gathered}
$$


Análisis de resistencia a la fractura de la cerámica de recubrimiento en la cresta marginal de coronas bilaminares de zirconia Y-TZP con infraestructura convencional y modificada: estudio in vitro

El estadístico de prueba:

$$
\mathrm{t}_{\mathrm{o}}=2.79
$$

Dado que $2.576>2.571$ se rechaza $H_{o}$.

Podemos decir que las coronas con estructura modificada soportan entre un $15 \%$ y $34 \%$ más de fuerza que las coronas con estructura convencional.

\section{Comparación de la Muestras en Fracaso de la Base}

Primero compararemos las muestras para una mejora del 15\% en relación con la estructura convencional.

Tabla 6 - Fracaso de la base en muestras convencional y modificada.

\begin{tabular}{cccccc}
\multicolumn{7}{c}{ VALOR DE CARGA DE FRACTURA (N) } \\
\hline & $\mathrm{n}$ & Media & $\begin{array}{c}\text { Desviación } \\
\text { estándar }\end{array}$ & Mínimo & Máximo \\
\hline CONVENCIONAL & 3 & 688.33 & 33.292 & 650 & 710 \\
MODIFICADA & 6 & 813.33 & 151.784 & 634 & 963 \\
\hline \hline
\end{tabular}

$\Delta_{15 \%}: 688.33 * 0.15=103.25$

$$
\begin{gathered}
H_{0}: \mu_{1}-\mu_{2} \leq-103.25 \\
H_{a}: \mu_{1}-\mu_{2}>-103.25 \\
\alpha=0.05(95 \% \text { de confianza })
\end{gathered}
$$

El estadístico de prueba:

$$
t_{\mathrm{o}}=-0.524
$$

Dado que $-0.524<2.365$ se acepta $\mathrm{H}_{o-}$

Segundo, compararemos las muestras para una mejora del $29 \%$ en relación com la

$$
\begin{aligned}
\Delta_{2996}: 611.3 * 0.29=177.27 & \\
& H_{0}: \mu_{1}-\mu_{2} \leq-177.27 \\
& H_{a}: \mu_{1}-\mu_{2}>-177.27 \\
\alpha & =0.05(95 \% \text { de confianza })
\end{aligned}
$$

El estadístico de prueba:

$$
\mathrm{t}_{\mathrm{o}}=2.242
$$

Dado que $2.242>2.101$ se rechaza $H_{o}$.

Segundo, compararemoms las muestras para una mejora del $33 \%$ en relación con la estructura convencional.

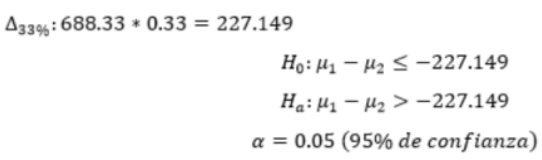

El estadístico de prueba:

$$
\mathrm{t}_{\mathrm{o}}=2.459
$$

Dado que $2.459>2.365$ se rechaza $H_{\text {o }}$.

Podemos decir que las coronas con estructura modificada soportan entre un $15 \%$ y $32 \%$ más de fuerza que laas coronas con estructura convencional.

\section{DISCUSIÓN}

Si bien los estudios in vitro no se asemejan en su totalidad a la realidad, son un parámetro sumamente importante para tomar decisiones en la práctica ya que pueden analizar factores que tienen influencia directa con el desempeño clínico. También pueden ser de alto valor al momento de estudiar y comparar factores específicos en un ambiente controlado.

En el presente estudio, todos los especímenes fueron fabricados y cementados sobre dados de trabajo de resina siguiendo todas las especificaciones para ser utilizados en una situación clínica real. La idea de estandarizar el espesor y forma de la cerámica de recubrimiento, así como los dos diseños de infraestructura, que se logran gracias al sistema CAD/CAM, y que son todos reproducidos de una manera exacta es esencial para poder realizar comparaciones de una manera confiable entre los diferentes grupos e identificar los factores de riesgo ${ }^{9-11}$.

Evidencias clínicas demuestran que la ubicación de los contactos oclusales tiene relevancia con el fracaso de las restauraciones. En una oclusión natural, los contactos incluyen cúspides, fosas y crestas marginales independientemente del esquema oclusal de Angle (Clase I, II, III). Los fracasos en esta zona son más propensos comparados con los de la fosa central y al parecer se debe a la mayor tensión al estrés especialmente en molares y premolares. Estos van a ser prácticamente imposibles de corregir con un procedimiento sencillo de pulido o reparado en boca por lo tanto se requerirá de un reemplazo de la restauración con sus consiguientes consecuencias. Es por esta razón, la decisión estratégica de haber puesto el indentador en esta zona específica de crestas marginales, para así con los resultados obtenidos en esta investigación saber si la infraestructura modificada soporta más cargas de fuerza que la infraestructura convencional ${ }^{2}$.

Cargas axiales estáticas hasta el fracaso son una excelente medida para comprobar la resistencia máxima de una restauración dental, sin embargo, estas no son las únicas fuerzas a las cuales estarán sometidas en una situación clínica real. Es por lo tanto que se deben considerar también los diferentes patrones de masticación, 
el arco antagonista, las características en donde las coronas van a ser cementadas ${ }^{2,12-13}$.

La cavidad oral es el ambiente en el cual se deben desenvolver éste tipo de restauraciones; es húmedo y con cambios constantes de temperatura, además existen fuerzas oclusales y para funcionales que exceden los $500 \mathrm{~N}$ y 1000 $\mathrm{N}$ respectivamente a las cuales deberán estar sometidas. Estas forman facetas de desgaste con micro porosidades que combinadas con el agua se transforman en una degradación mecánica y química (LTD, Low Thermal Degradation) con fisuras internas que terminan en fractura ${ }^{2-3,14}$.

Pruebas de cargas cíclicas térmicas en combinación con agua pueden también degradar a la cerámica, especialmente a las cerámicas vítreas utilizadas para el recubrimiento de las coronas. Estas pruebas de fatiga tienen relevancia también a la hora de hacer estudios del comportamiento de los diferentes tipos de coronas ya que proveen de situaciones clínicas de masticación más reales ${ }^{15}$.

Sin embargo, el propósito principal de este estudio in vitro fue identificar cual es la carga máxima de resistencia a la fractura que va a soportar la cerámica de recubrimiento y para ello, la prueba que se debe utilizar es la de cargas estáticas axiales hasta el fracaso, debido a que especialmente en el sector posterior es donde ocurrirá la mayor cantidad de fuerzas que deberán soportar las restauraciones.

En este estudio, el universo de muestras fue de 10 coronas con infraestructura convencional y 10 coronas con infraestructura modificada; si bien es un tamaño pequeño de muestras, esto se debió al alto costo y tiempo que representó la fabricación de las mismas ya que fueron elaboradas con materiales de primera calidad producidos en Japón por una marca con una trayectoria de seriedad de muchos años.

La producción fue hecha en un laboratorio altamente calificado y con tecnología de punta con el propósito de obtener muestras exactas para poder ser utilizadas en una situación clínica real y poder ser comparados los resultados estadísticos entre los dos grupos.

Es bien entendido que la cerámica es un vidrio frágil y que necesita de un buen soporte para elevar su coeficiente de resistencia a la fractura. Por lo tanto, un diseño inadecuado de infraestructura no va a contribuir con el objetivo anteriormente mencionado a la hora de aplicar cargas de fuerzas oclusales.

La presente investigación in vitro fue basada en un estudio previo ${ }^{2}$ en el cual presenta un diseño de infraestructura modificada que va a dar más soporte a la cerámica de recubrimiento en la cara lingual e interproximal la cual es justamente donde se ubican las crestas marginales.

La información que se obtuvo en el presente estudio nos permite decir que existe un mejor desempeño de las coronas bilaminares de zirconio con infraestructura modificada en los cuatro modos de fracaso encontrados.
Las muestras con infraestructura modificada poseen rangos intercuartil menores a las muestras convencionales. Por lo tanto, el comportamiento de las coronas con infraestructura modificada posee una mayor homogeneidad que las coronas con infraestructura convencional.

Se observó también que los datos atípicos de las muestras convencionales tienden a elevar la media de carga de fuerza para la fractura, mientras que los datos atípicos de las muestras modificadas no generan este fenómeno de una manera considerable.

Si bien en el análisis del modo de fracaso adhesivo los datos indican que las muestras con infraestructura modificada poseen una media menor que las muestras convencionales, la desviación estándar y el posicionamiento de los datos nos permiten afirmar que las muestras modificadas poseen una mayor estabilidad $\mathrm{y}$ mejor desempeño.

La fuerza de carga axial que se debe ejercer para provocar un fracaso en las coronas con infraestructura modificada fue mayor que la fuerza necesaria para las coronas con infraestructura convencional. Se deduce que esto se debe al mayor soporte que brinda la infraestructura modificada a la cerámica de recubrimiento. Debido a ello encontramos también un mayor número de fracasos en la base.

En cuanto al fracaso de la infraestructura, como se mencionó anteriormente, el universo de muestras para este análisis no contiene la suficiente cantidad de datos para hacer un estudio estadístico al respecto.

En función del valor de carga para generar una fractura, podemos decir que a través de este estudio los datos arrojaron que las coronas bilaminares de zirconia con infraestructura modificada, soportan entre un $15 \%$ y $28 \%$ más de fuerza que las coronas bilaminares con infraestructura convencional.

En el modo de fracaso cohesivo, los datos arrojaron que las coronas bilaminares de zirconia con infraestructura modificada soportan entre un $15 \%$ y $34 \%$ más que las coronas bilaminares de zirconia con infraestructura convencional.

En el modo de fracaso adhesivo, los datos arrojaron que las muestras modificadas soportan entre un 15\% y 34\% más que las muestras convencionales.

Para que exista un modo de fracaso de la base, los datos arrojaron que las muestras modificadas soportaron entre un $15 \%$ y $32 \%$ más que las muestras convencionales.

$\mathrm{Si}$ bien los resultados de este estudio in vitro no concuerdan con otros previos publicados, en cuanto a la resistencia a la fractura de la cerámica de recubrimiento ya que los promedios de los datos de los dos tipos de muestras utilizados para esta investigación son menores ${ }^{2,5,11,15}$ si hay concordancia en cuanto a que las muestras con infraestructura modificada tienen en promedio una resistencia más elevada en los cuatro modos de fractura encontrados que las muestras con infraestructura 
Análisis de resistencia a la fractura de la cerámica de recubrimiento en la cresta marginal de coronas bilaminares de zirconia Y-TZP con infraestructura convencional y modificada: estudio in vitro

convencional ${ }^{2}$. Esto se debe al mayor soporte que esta provee a la cerámica de recubrimiento volviéndolas más confiables para uso en casos clínicos reales.

Una diferencia importante con estudios previos publicados, excepto el realizado ${ }^{13}$ el cual no lo realiza con cargas estáticas axiales si no con cargas cíclicas de fatiga, es que para esta investigación se colocó al indentador esférico metálico de $3.7 \mathrm{~mm}$ de diámetro en la cresta marginal del primer molar inferior derecho en vez de colocarlo en el centro de la corona o de una cúspide. Esto probablemente trajo como consecuencia valores inferiores de resistencia a la fractura de la cerámica de recubrimiento en los dos tipos de muestras ya que es la zona adyacente al margen ${ }^{16-17}$.

Además, el tamaño relativamente pequeño del diámetro del indentador y al ser metálico puede derivar en valores de carga de fractura inferiores ${ }^{11}$.

\section{CONCLUSIÓN}

Recordando que la hipótesis nula propuesta para este estudio es que, el valor medio de fuerza de carga axial estática (N) necesario para provocar la fractura de la cerámica de recubrimiento de las coronas con infraestructura modificada es al menos el 15\% más alta que la media de fuerza de carga axial estática para provocar la fractura de la cerámica de recubrimiento de las coronas con infraestructura convencional; en todos los modos de fracaso la hipótesis nula fue aceptada.

Es interesante e importante mencionar que además de que la hipótesis nula fue aceptada en el presente estudio, los valores de mejora de resistencia a la fractura suben desde un $28 \%$ hasta un $34 \%$ dependiendo de cada modo de fracaso analizado, lo cual podemos considerar que son valores bastante significativos.

Este estudio se basó en fuerzas estáticas axiales para el fracaso, sin embargo, se debe entender que estas no son solamente el único tipo de fuerzas a las que este tipo de restauraciones van a ser sometidas en una situación clínica real como son las fuerzas laterales que producen fatiga del material o la degradación térmica o mecánica por inclusión de agua. Existen también factores como el desgaste que producen los instrumentos rotatorios al momento de efectuar ajustes en la oclusión y el tipo de antagonista que generan facetas de desgaste las cuales no estuvieron presentes en esta investigación por ser in vitro. Parámetros que deben ser tomados en cuenta para futuras investigaciones, así como también incrementar el número de muestras a ser analizadas para poder tener una información estadística más precisa.

\section{REFERENCIAS}

1. Hamza TA, Sherif RM. Fracture resistance of monolithic glassceramic versus bilayered zirconia-based restorations. J Prosthodont. 2019;28(1):e259-64.

2. Silva NRFA, Bonfante EA, Rafferty BT, Zavanelli RA, Rekow ED, Thompson VP, et al. Modified Y-TZP core design improves allceramic crown reliability. J Dent Res. 2011;90(1):104-8.

3. Agustín-Panadero R, Román-Rodríguez JL, Ferreiroa A, Solá-Ruiz MF, Fons-Font A. Zirconia in fixed prothesis. A literature review. J Clin Exp Dent. 2014;6(1):66-73.

4. Guess PC, Schultheis S, Bonfante EA, Coelho PG, Ferencz JL, Silva NRFA, et al. All ceramic systems: laboratory and clinical performance. Dent Clin North Am. 2011;55(2):333-52.

5. Guess PC, Bonfante EA, Silva NRFA, Coelho PG, Thompson VP. Effect of core design and veneering technique on damage and reliability of Y-TZP supported crowns. Dent Mater. 2013;29(3):307-16.

6. Rekow ED, Silva NRFA, Coelho PG, Zhang Y, Guess P, Thompson VP, et al. Performance of dental ceramics: challenges for improvements. J Dent Res. 2011;90(8):937-52.

7. Shahin A, Al-Wahadni A, Masri R. Zirconia-based restorations: literature review. Int J Med Res Prof. 2017;3(2):253-60.

8. Tang YL, Kim JH, Shim JS, Kim S. The effect of different cooling rates and coping thickness on the failure load of zirconiaceramic crowns after fatigue loading. J Adv Prosthodont. 2017;9(3):152-8.

9. Pádua JM, Teles RF. Preparos protéticos em dentes posteriors para Sistemas CAD/CAM. In: CAD/CAM No Laboratorio e na clínica a odontologia digital. Nova Odessa: Napoleão; 2017.

10. Soares LM, Soares C, Miranda ME, Basting RT. Influence of coreveneer thickness ratio on the fracture load and failure mode of zirconia crowns. J Prosthodont. 2019;28(2):209-15.

11. Montgomery DC, Runger GC. Probabilidad y estadística aplicadas a la ingeniería. 2nd ed. México: Limusa Wiley; 2005.

12. Al-Wahadny A, Shahin A, Kurtz K. Veneered zirconia-based restorations fracture resistance analysis. J Prosthodont. 2018;27(7):651-8.

13. Fardin VP, de Paula VG, Bonfante EA, Coelho PG, Bonfante G. Lifetime prediction of zirconia and metal ceramic crowns loaded on marginal ridges. Dent Mater. 2016;32(12):1543-54.

14. Choi S, Yoon HI, Park EJ. Load-bearing capacity of various $\mathrm{CAD} / \mathrm{CAM}$ monolithic molar crowns under recommended occlusal thickness and reduced occlusal thickness conditions. J Adv Prosthodont. 2017;9(6):423-31.

15. Larsson C, Madhoun SE, Wennerberg A, Vult von Steyern P. Fracture strength of yttria-stabilized tetragonal zirconia polycrystals crowns with different design: an in vitro study. Clin Oral Implantol Res. 2012;23(7):820-6.

16. Zhang Y, Lawn BR. Novel zirconia materials in dentistry. J Dent Res. 2018;97(2):140-7.

17. Preis V, Letsch C, Handel G, Behr M, Schneider-Feyrer S, Rosentritt $\mathrm{M}$, et al. Influence of substructure design, veneer application technique, and firing regime on the in vitro performance of molar zirconia crowns. Dent Mater. 2013;29(7):e113-21. 